\title{
Structural Analysis of Multiple Lab-Evolved Proteins that Bind HIV-1 TAR RNA with NanoMolar Affinity
}

S. Shashank Chavali§, Ivan A. Belashov§, David Crawford ${ }^{\prime \prime l}$, Brian R. McNaughton $\psi$, and Joseph E. Wedekind\$

§Dept. Biochemistry \& Biophysics and Center for RNA Biology, University of Rochester School of Medicine and Dentistry, Rochester NY 14642, USA

${ }$ Department of Chemistry and IDepartment of Biochemistry \& Molecular Biology, Colorado State University, Fort Collins CO 80523, USA

Innovative approaches are needed to identify new therapeutics that target HIV-1, especially those that act on novel aspects of the viral lifecycle. HIV-1 TAR (trans activation response element) RNA is a validated drug target that resists mutations to maintain essential interactions with the viral protein Tat and the host pTEFb complex. So far, TAR has evaded discovery of compounds with sufficient affinity and selectivity to warrant pharmaceutical development. To meet this challenge, we undertook a 'molecular exapation' approach that used lab-based evolution to select numerous high-affinity IAR Binding Proteins (TBPs) derived from RRM1 (RNA recognition motif 1) of the U1A spliceosomal protein. Here I describe the lab-evolution process and present isothermal titration calorimetry (ITC) analyses of four family members that differ in the amino acid compositions in the evolved $\beta 2-\beta 3$ loop region and C-terminus. Remarkably, small changes produce a range of affinities: TBP6. 6 exhibits a $K_{D}$ of $1.6 \pm 0.3 \mathrm{nM}$; TBP6.7 exhibits a $K_{\mathrm{D}}$ of $2.5 \pm 0.6 \mathrm{nM}$; TBP6.9 exhibits a $K_{\mathrm{D}}$ of $2.6 \pm 0.78 \mathrm{nM}$; and TBP6.11 at 37 ${ }^{\circ} \mathrm{C}$ exhibits a $K_{\mathrm{D}}$ of $14.1 \pm 1 \mathrm{nM}$. Each TBP has a distinct thermodynamic signature, suggesting that TBPs may use different modes of TAR readout. To test this hypothesis, we determined the crystal structures of TBP6.6 and TBP6.7. Although both proteins use a similar mode of $\beta 2-\beta 3$ loop recognition in which arginines read conserved guanine bases in the TAR major groove, distinct differences are apparent in the modes of loop stabilization and the protein backbone. Overall, we hypothesize that cyclic peptides comprising minimal TBP $\beta 2-\beta 3$ loops will serve as entry points into the production of novel therapeutics that inhibit HIV-1 transcription. 